\title{
Re: Safety of Ultrasound-Guided Transrectal Extended Prostate Biopsy in Patients Receiving Low-Dose Aspirin
}

\author{
Ioannis Kariotis, Prodromos Philippou, Demetrios Volanis, Efraim Serafetinides, Demetrios \\ Delakas
}

Department of Urology, “Asklipieion” General Hospital, Athens, Greece

Int Braz J Urol. 2010; 36: 308-16

To the Editor,

Clinicians in general should note procedures that may appear to be associated with a high or low risk of bleeding, despite the lack of high quality methodological scientific evidence in this area. Furthermore, there are invasive procedures which possess different risks of bleeding, as well as patients with different risks of thrombosis. Additionally, clinicians must consider the existence of various ways of perioperative anticoagulation and adequate identification for those with "high risk" for antithrombotic cessation afterwards.

Studies dealing with safety of minor invasive procedures on patients taking into consideration antithrombotics in general are not abundant in the literature, which has often raised practical concerns and controversies regarding how to manage them appropriately, mainly in perioperative intervention. These interventions with minor risks have usually been undertaken using exaggerated caution or individualized according to the urologist's experience.

In order to give some examples of this, the following are cited:

1. Resection of colonic polyps, especially sessile polyps $>2 \mathrm{~cm}$ in diameter, in which bleeding may occur at the transected stalk;

2. Cardiac pacemaker or defibrillator implantation, in which separation of the infraclavicular fascial layers and lack of good hemostasis of unopposed tissues within the pacemaker or defibrillator pocket may be predisposed to hematoma development and serious complications.
3. Biopsy of the prostate or kidney, in which the presence of highly vascular tissue and endogenous urokinase may promote post-biopsy bleeding $(1,2)$.

The study carried out by Kariotis et al. emphasized that the previous use of aspirin does not necessarily mean it has been withdrawn. The action to maintain drugs such as oral anticoagulation or even antiagregation has great relevance and was already considered among other authors(3-5), generating matter for debate $(3,6)$.

It is important to realize that nowadays, more patients that are elderly will be referred to undergo a prostate biopsy. Also, the majority of patients will coexist with cardiac diseases, such as coronary stents, enlarged atriums with complex arrhythmias, valve problems, severe arterial vasculopathy, or venous thrombosis among complex comorbities; all requiring adequate anticoagulation or antiagregation in order to avoid severe or disastrous thromboembolic events. Based on these assumptions, clinicians have always to outweigh risks and benefits. Their ultimate decision on whether to withdraw or withhold these antithrombotics is still a matter of judgment, preferences and values as well as the type and amount of biopsies undertaken.

In the same way, Lhezue et al. (2) already described similar findings in 2005, but instead of administering aspirin, the concomitant use of oral anticoagulant (warfarin), a stronger anticoagulant, 
was used. Also, they concluded that its suspension, in order to perform prostate biopsy, should not be necessary according to their favorable outcome and absence of complications.

More recently, a prospective randomized trial was undergone in order to determine whether the incidence and duration of bleeding complications after transrectal prostate biopsy in patients not discontinuing low-dose aspirin are greater than in those discontinuing it (4).

Despite the discussion regarding whether the number of biopsy cores taken (between 4 and 9) would be considered the best practice, physicians have to conjugate or balance its context of realization.

In addition, among all controversies still present in our practice, the need to avoid a thromboembolic event, such as embolic stroke or intracoronary stent thrombosis, will dominate perioperative antithrombotic management, irrespective of bleeding risk. aspects:

Lastly, it is important to highlight 3 important

1. Resuming antithrombotic therapy after a surgical or invasive procedure, it takes 2 to 3 days for an anticoagulant effect to begin after the administration of warfarin. Whereas it takes minutes for an antiplatelet effect to begin after the administration of aspirin, and 3 to 7 days for peak inhibition of platelet aggregation to be reached after the application of a $(75 \mathrm{mg})$ maintenance dose of clopidogrel (1).

2. The majority of surgical or other invasive procedures are being performed without hospitalization or with a short hospital stay; consequently, potential thromboembolic or bleeding-related complications are likely to occur while the patient is at home. Close follow-up of patients during the early period after a procedure is, therefore, warranted to allow early detection and expedited treatment of potential complications.

3. Studies are lacking with regard to patients who are receiving clopidogrel and require a prostate biopsy, although it is probable that the continuation of clopidogrel and aspirin in patients undergoing minor procedures will increase the risk of bleeding as mentioned above that seen with the use of aspirin alone.

\section{REFERENCES}

1. Douketis JD, Berger PB, Dunn AS, Jaffer AK, Spyropoulos $\mathrm{AC}$, Becker $\mathrm{RC}$, et al.: The perioperative management of antithrombotic therapy: American College of Chest Physicians Evidence-Based Clinical Practice Guidelines (8th Edition). Chest. 2008; $133(6$ Suppl): 299S-339S.

2. Ihezue CU, Smart J, Dewbury KC, Mehta R, Burgess L: Biopsy of the prostate guided by transrectal ultrasound: relation between warfarin use and incidence of bleeding complications. Clin Radiol. 2005; 60: 459-63; discussion 457-8.

3. Halliwell OT, Lane C, Dewbury KC: Transrectal ultrasound-guided biopsy of the prostate: should warfarin be stopped before the procedure? Incidence of bleeding in a further 50 patients. Clin Radiol. 2006; 61: 10689.

4. Giannarini G, Mogorovich A, Valent F, Morelli G, De Maria M, Manassero F, et al.: Continuing or discontinuing low-dose aspirin before transrectal prostate biopsy: results of a prospective randomized trial. Urology. 2007; 70: 501-5.

5. Halliwell OT, Yadegafar G, Lane C, Dewbury KC: Transrectal ultrasound-guided biopsy of the prostate: aspirin increases the incidence of minor bleeding complications. Clin Radiol. 2008; 63: 557-61.

6. Giannarini G, Mogorovich A, Selli C: Re: transrectal ultrasound-guided biopsy of the prostate: aspirin increases the incidence of minor bleeding complications. Clin Radiol. 2008; 63: 1386-7; author reply 1387.

Dr. Rubens Costa Filho Chair, Intensive Care Unit Pro-Cardiaco Hospital

Rio de Janeiro, RJ, Brazil

E-mail: rubens1956@gmail.com 